\title{
IOWA STATE UNIVERSITY
}

\begin{tabular}{|c||}
\hline \hline INCENTIVES AND OUTCOMES IN A STRATEGIC \\
SETTING: THE 3-POINTS-FOR-A-WIN SYSTEM IN \\
SOCCER \\
GianCarlo Moschini \\
June 2008 \\
Working Paper \# 08021 \\
\hline Department of Economics \\
Working Papers Series
\end{tabular}

Ames, Iowa 50011

Iowa State University does not discriminate on the basis of race, color, age, religion, national origin, sexual orientation, gender identity, sex, marital status, disability, or status as a U.S. veteran. Inquiries can be directed to the Director of Equal Opportunity and Diversity, 3680 Beardshear Hall, (515) $294-7612$. 


\title{
InCENTIVES AND OUTCOMES IN A STRATEgIC SETTING: THE 3-PoINTS-FOR-A-WIN SYSTEM IN SOCCER
}

GianCarlo Moschini

\begin{abstract}
I exploit a major structural change that has occurred in world soccer to study the impact of incentives on outcomes in a strategic setting. A game-theoretic model is developed that captures some essential strategic elements of soccer vis-à-vis the number of points awarded to a win. The observable implications of the model are tested using a large dataset that spans 30 years and 35 countries. The empirical results support the theoretical model and show that the 3-point system has led to a statistically significant increase in the expected number of goals and a decrease in the fractions of drawn matches.
\end{abstract}

Key Words: Association football, Nash equilibrium, panel data, strategic incentives, supermodularity, tournaments.

JEL codes: C72, L83, C23.

June 2008

GianCarlo Moschini is Professor and Pioneer Chair in Science and Technology Policy, Department of Economics, Iowa State University, Ames, IA 50011. Phone: (515)294-5761. Fax: (515)294-6336. E-mail: moschini@,iastate.edu.

The author is grateful to Xin Pan for his expert research assistance in assembling the data set and would like to thank Harvey Lapan for commenting on an earlier draft.

This is Working Paper \# 08021, Department of Economics, Iowa State University. 


\section{INTRODUCTION}

The behavior of agents in a strategic contest is a central concern of economic models, and the notion of Nash equilibrium (and various extensions and refinements thereof) is the guiding principle of analysis. Results in this setting are often sensitive to the structure of the interaction, and taking models' predictions to an empirical test is a notoriously difficult task. Contests are the quintessential characteristic of sport, and thus sport situations share a number of features with economic problems that arise in many other contexts. Because sport competitions have clear-cut rules, competitors are strongly motivated, and relevant data is often observable, sport settings are proving to be a fruitful area of empirical inquiry to test important tenets of economic models. For example, the notion of Nash equilibrium has been put to a direct test by using data on serve choices in tennis matches in Walker and Wooders (2001), and Hsu, Huang and Tang (2007); soccer penalty kicks data in Chiappori, Levitt and Groseclose (2002) and Palacios-Huerta (2003); and near-post, far-post soccer goals in Moschini (2004). The results of these studies are both interesting and encouraging and broadly support the notion that players' choices are in accord with equilibrium predictions.

In this article I propose to use soccer (association football) data in a new direction, to investigate the impact of a major structural change in the organization of soccer competitions that took place in the mid-1990s. Most national soccer competitions are typically organized as double round-robin tournaments in which every team plays all others in its league twice (at home and away). Points earned in every match are added together, and rewards are assigned according to the totals earned in the entire competition. ${ }^{1}$ Unlike some other sports, drawn matches are common in soccer, and the point system determines their importance relative to victories. The traditional way to handle that was the 2-1-0 system whereby a tie was worth half as much as a victory (and a loss was worth nothing). England first replaced that with a 3-1-0 system (3 points for a win and 1 point for a tie) starting in the $1981 / 82$ season in an effort to promote a more attacking brand of soccer. A few other countries followed suit in the years that followed, but the new system became widespread

\footnotetext{
${ }^{1}$ The chief reward is the championship title, but the final standing carries other rewards and penalties. In most countries, teams that finish close to the top may qualify for other competitions (e.g., Champions League or UEFA cup in Europe). Also, because most national soccer leagues have membership through promotion and relegation (Noll, 2003), teams that finish at the bottom are penalized through relegation to the next lower league.
} 
after it was embraced by FIFA (Fédération Internationale de Football Association), the game's world governing body, to structure the initial (round-robin) phase of the 1994 world cup. In the years that followed, virtually every country adopted the new 3-point system.

The widespread and systematic introduction of the 3-point system in soccer provides an interesting natural experiment to investigate the effects of changing rewards and incentives on outcomes in a tournament setting. Did the increased weight given to victories translate into more attacking play and goals, as per the hopeful expectation? And is that really what one should expect from such a structural change anyway? A few studies that have investigated such questions have stressed some negative results. Brocas and Carrillo (2004) develop a conceptual model of a soccer match as a two-stage game and cast doubts on the expectation that the rule change in question necessarily induces more attack. Their main point is that the new system, while encouraging more attack towards the end of the game, may also induce teams to play more defensively at the beginning of the game. Correira Guedes and Machado (2002) similarly note possible unintended effects of the point system change due to asymmetric abilities (underdogs facing rivals of superior abilities may actually reduce their level of offense). Garicano and Palacios-Huerta (2005) emphasize the added incentive for sabotage tactics that the new point system may induce.

In this article I reconsider the question of whether the introduction of the 3-1-0 point system in soccer actually has had significant effects. I start by developing an explicit game-theoretic model of soccer wherein the number of points awarded to a win has a meaningful role. When a team's strategic choice applies for the entire soccer match, the model emphasizes the supermodular nature of the resulting game. This means that strong comparative statics results are possible, and they carry some testable observable implications. I then argue that such implications are likely to be robust to the more general view of a soccer match as a multi-stage game. Because supermodularity is lost in this multi-stage setting, the argument proceeds by considering in some detail the two-stage context suggested by Brocas and Carrillo (2004), and by a computational analysis of a fairly general parametric specification of the model. Next I test the observable implications of the model empirically by utilizing an extensive and original dataset that spans 30 years and 35 countries. The empirical results are supportive of the implications of the theoretical model. I find that, overall, the 3-point system has led to a statistically significant increase in the expected 
number of goals and a statistically significant decrease in the fractions of matches that end in a draw.

\section{THE MODEL}

Models of sporting contests often emphasize the allocation-of-effort problem, in response to the incentive structure, as suggested by the study of tournaments originated by Lazear and Rosen (1981). ${ }^{2}$ In our context, however, I find it more appealing to proceed differently by taking a team's amount of effort as given and focusing instead on the allocation of this effort to attacking and/or defensive purposes. This is consistent with the empirical finding of Ferrall and Smith (1999) who study sport championship series and find that teams play as well as possible in a given game, regardless of its strategic importance in the series. Indeed, the intended aim of the 3-point system change - to encourage more attacking and less defensive play_-speaks directly to the choice of effort type. What typically holds teams back from a more attacking strategy is not the cost of efforts involved but its risky attribute: a more attacking stance not only increases a team's chances of scoring a goal but also increases the prospects of a successful counter-attack by the opposing team and thus increases the chances of conceding a goal. The setting here is not unlike typical R\&D problems in the innovation literature, for which, in addition to the problem of choosing the amount of $\mathrm{R} \& \mathrm{D}$, a critical question concerns the type of R\&D projects to pursue, as in Dasgupta and Maskin (1987). Insofar as playing with a more attacking strategy is riskier, the problem is also akin to the strategic choice of variance discussed by Cabral (2003).

The model presumes that the teams are engaged in a long championship (usually a double round-robin, as noted earlier) with the winner determined by the highest total number of points. It is therefore maintained that, in any one match, each team's objective is to maximize the expected number of points in that match. The two teams that compete in any one match are labeled $A$ and $B$. As in Brocas and Carrillo (2004), each team is assumed to choose an action $a_{i} \in[0,1], i \in\{A, B\}$, that is taken to represent the degree of attacking/defensive play (e.g., the choice of team "formation," that is, how many defenders, midfielders and forwards are fielded and how they are positioned on the pitch). Specifically, a more attacking stance attains as $a_{i} \rightarrow 1$, and a more defensive stance results as $a_{i} \rightarrow 0$.

\footnotetext{
${ }^{2}$ Szymanski (2003) and Frick (2003) provide comprehensive reviews of this and related
} issues. 
The actions chosen by the two teams affect the probability of goals being scored. Naturally I assume that a more offensive play by team $i\left(a_{i} \rightarrow 1\right)$ increases the probability that this team scores. But because this choice necessarily entails a less defensive posture, it also increases the probability that the other team scores. Conversely, a more defensive choice for team $i\left(a_{i} \rightarrow 0\right)$ reduces both teams' probability of scoring. Because for the time being the match is modeled as a one-stage game, I work directly with the probability that each team scores one goal or more than the other team. Specifically, $p_{i}\left(a_{i}, a_{j}\right)$ represents the probability that team $i$ scores more goals than the other team $(i, j \in\{A, B\}, j \neq i)$, i.e., that team $i$ wins the match.

The expected number of points for team $i$, for a given action profile $\left(a_{i}, a_{j}\right)$, is

$$
p_{i}\left(a_{i}, a_{j}\right) \cdot n+p_{j}\left(a_{j}, a_{i}\right) \cdot 0+\left[1-p_{i}\left(a_{i}, a_{j}\right)-p_{j}\left(a_{j}, a_{i}\right)\right] \cdot 1
$$

where $n$ is the number of points awarded to a win (thus, $n=2$ or $n=3$ ), and, as discussed, a loss (which for team $i$ occurs with probability $p_{j}\left(a_{j}, a_{i}\right)$ ) is awarded zero points, and a draw (which occurs with probability $\left.1-p_{i}\left(a_{i}, a_{j}\right)-p_{j}\left(a_{j}, a_{i}\right)\right)$ is awarded one point. The teams' payoffs can therefore be written as

$$
U_{i}\left(a_{i}, a_{j} ; n\right)=1+(n-1) p_{i}\left(a_{i}, a_{j}\right)-p_{j}\left(a_{j}, a_{i}\right), \quad i, j \in\{A, B\}, j \neq i
$$

The characterization of equilibrium clearly will depend heavily on the properties of the probability functions $p_{i}\left(a_{i}, a_{j}\right)$. I make the following explicit assumptions on the scoring probability functions:

ASSUMPTION 1. For all $\left(a_{i}, a_{j}\right) \in[0,1] \times[0,1]$ the probability functions $p_{i}\left(a_{i}, a_{j}\right)$, $i, j=A, B(j \neq i)$, satisfy
(a) $\partial p_{i}\left(a_{i}, a_{j}\right) / \partial a_{i} \geq 0$
(b) $\partial p_{i}\left(a_{i}, a_{j}\right) / \partial a_{j} \geq 0$
(c) $\quad \partial^{2} p_{i}\left(a_{i}, a_{j}\right) / \partial a_{i}^{2} \leq 0$ 
(d) $\quad \partial^{2} p_{i}\left(a_{i}, a_{j}\right) / \partial a_{j}^{2} \geq 0$

(e) $\quad \partial^{2} p_{i}\left(a_{i}, a_{j}\right) / \partial a_{j} \partial a_{i} \geq 0$

(f) $\quad \partial^{2} p_{i}\left(a_{i}, a_{j}\right) / \partial a_{j} \partial a_{i}=\partial^{2} p_{j}\left(a_{j}, a_{i}\right) / \partial a_{i} \partial a_{j}$

where the inequalities in (a)-(c) hold strictly on $\left(a_{i}, a_{j}\right) \in[0,1) \times[0,1)$.

Hence, Assumptions 1(a) and 1(c) say that the own-scoring probability is an increasing and concave function of the own degree of offensive play. Assumptions 1(b) and 1(d) reflect the presumption that a team's offensive play also increases the other team's scoring possibilities and that this relation is convex. Assumption 1(e) maintains that the teams' attacking choices are complementary as far as the probability of producing goals is concerned (i.e., the own marginal productivity of offensive play is not decreased by the other team's offensive choice). Assumption 1(f) maintains a degree of symmetry in the probability function (but note that I do not assume that the probability functions are the same for the two teams). ${ }^{3}$

The structure of the model developed in the foregoing allows us to claim the following strong results.

Proposition 1. Given the foregoing model of a soccer match, a Nash equilibrium in pure strategies exists, and its comparative statics entail that an increase in the number of points awarded to a victory, from $n=2$ to $n=3$, increases in the attacking choices of both teams.

The proof of this proposition follows immediately from the observation that ours is a particular instance of a supermodular game, a class of contests with strategic complementarities that has attracted considerably theoretical interest (see Vives, 2005, for a comprehensive review). The hallmark of a supermodular game is that of having payoff functions that display strategic complementarities both in own strategies and in the strategies of rivals. In the one-dimensional action space of interest here, this boils down to payoff

\footnotetext{
${ }^{3}$ Assumptions 1(e) and 1(f) are somewhat more general than the corresponding ones in Brocas and Carillo (2004). In particular, I do not assume $p_{A}\left(a^{\prime}, a^{\prime \prime}\right)=p_{B}\left(a^{\prime \prime}, a^{\prime}\right)$, $\forall a^{\prime \prime}, a^{\prime} \in[0,1]$, and thus the two teams are allowed to have different strengths.
} 
functions $U_{i}\left(a_{i}, a_{j} ; n\right)$ that possess increasing differences in $\left(a_{i}, a_{j}\right)$, a condition that, in our smooth formulation, is equivalent to $\partial^{2} U_{i} / \partial a_{j} \partial a_{i} \geq 0$. Differentiating (1), in view of Assumption 1(f), yields

$$
\partial^{2} U_{i}\left(a_{i}, a_{j} ; n\right) / \partial a_{j} \partial a_{i}=(n-2)\left[\partial^{2} p_{i}\left(a_{i}, a_{j}\right) / \partial a_{j} \partial a_{i}\right]
$$

and thus, given Assumption $1(\mathrm{e}), \partial^{2} U_{i} / \partial a_{j} \partial a_{i} \geq 0$ as long as $n \geq 2$. Thus, for our setting in which either $n=2$ or $n=3$, the teams' payoff functions display strategic complementarities.

Existence of pure-strategy Nash equilibria, in a more general setting that includes our model as a special case, was established by Topkis (1979). The generality of that result stems from the fact that both existence of equilibrium and comparative statics results rely simply on order structure and monotonicity properties, such as the notion of increasing differences (Vives, 1990). The key comparative statics result of Proposition 1 claims that the Nash equilibrium solution $a_{i}^{*}$ satisfies $\partial a_{i}^{*} / \partial n \geq 0(i \in\{A, B\})$. That is, increasing the number of points awarded to a win in this game should unambiguously increase the teams' equilibrium attacking choices. The result is predicated upon the teams' payoff functions $U_{i}\left(a_{i}, a_{j} ; n\right)$ displaying increasing differences in $\left(a_{i}, n\right)(i \in\{A, B\})$. That this property applies is readily established by differentiating the payoffs of equation (1), which yields

$$
\partial^{2} U_{i} / \partial a_{i} \partial n=\partial p_{i}\left(a_{i}, a_{j}\right) / \partial a_{i} \geq 0
$$

where the inequality (which holds strictly in the interior of the action set) follows from Assumption 1(a).

Thus, the natural supermodular structure of the model at hand yields strong and unambiguous comparative statics results on the effects of the policy rule $n$ on the strategic choices of the contestants. Because the teams' attacking choices are not directly observable, to test such predictions we need to translate their implications into observable outcomes. To that end, I focus on two statistics, the probability that a match ends in a draw $z\left(a_{i}, a_{j}\right)$ and the expected number of goals $y\left(a_{i}, a_{j}\right)$. Given the structure of our model, the probability 
that the match ends in a draw satisfies $z\left(a_{i}, a_{j}\right) \equiv 1-p_{i}\left(a_{i}, a_{j}\right)-p_{j}\left(a_{i}, a_{j}\right)$. Hence, if the 3point rule increases the teams' attacking choices as per Proposition 1, then, because of the monotonicity of scoring probability functions in the contestants' actions, it follows that the probability that a match ends in a draw must decrease.

To establish unambiguously a link between Proposition 1 and the expected number of goals, on the other hand, a bit more structure is required. The difficulty is that, in our formulation, the function $p_{i}\left(a_{i}, a_{j}\right)$ denotes the probability that team $i$ scores more goals than the other team, with the number of scored goals being unspecified. A clear-cut result is possible if we re-interpret the model by assuming that at most one goal is scored by either team. In such a case, the expected number of goals in a match satisfies $y\left(a_{i}, a_{j}\right)=p_{i}\left(a_{i}, a_{j}\right)+p_{j}\left(a_{i}, a_{j}\right)$. Because of the monotonicity of scoring probability functions in the contestants' actions, it follows that the expected number of goals $y\left(a_{i}, a_{j}\right)$ is monotonically increasing in actions. I summarize the foregoing as follows.

Proposition 2. In the model of a soccer match developed in this article, an increase in the number of points awarded to a victory, from $n=2$ to $n=3$, leads to (a) a decrease in the probability that a match ends in a draw; and (b) an increase in the expected number of goals in a match, provided that $p_{i}\left(a_{i}, a_{j}\right)$ is interpreted as the probability of team $i$ scoring one goal when the other team does not $(i, j \in\{A, B\}, j \neq i)$.

Alternatively, the issue of expected number of goals can be addressed more explicitly by recasting the soccer match as a multi-stage game, under the assumption that at most one goal can be scored by either contestant in any one stage. Such an extension, however, also raises the issue of whether the teams' actions ought to be allowed to adjust to the "state" of the game, an issue that I discuss next after considering the following illustration.

\section{A. Example I}

A simple parameterization that fits the structure and assumptions laid out in the foregoing is 


$$
p_{i}=\gamma\left[\left(1+\beta_{i}-\beta_{j}\right)\left(a_{i}-a_{i}^{2} / 2\right)+a_{j}^{2} / 2\right], \quad i, j=A, B ; j \neq i
$$

where $\gamma \in(0,1 / 2)$ is a scaling parameter that ensures that actions are mapped into the admissible probability space $[0,1]$, and $\beta_{i}, \beta_{j} \in(0,1)$ are parameters that index each team's strength. The restriction on the $\gamma$ parameter follows by observing that probabilities are increasing in either action, and $p_{i}+p_{j}=2 \gamma$ at $a_{i}=a_{j}=1$. With this parameterization, in fact, we have $\partial^{2} U_{i} / \partial a_{j} \partial a_{i}=0$, implying that a team's optimal action is actually independent of the other team's action. Upon solving for the optimal action choice one finds

$$
a_{i}^{*}=(n-1)\left(1+\beta_{i}-\beta_{j}\right) /\left[1+(n-1)\left(1+\beta_{i}-\beta_{j}\right)\right], \quad i, j=A, B ; j \neq i .
$$

Thus, $a_{i}^{*}$ is strictly increasing in the number of points awarded to a victory. For instance, if teams were of equal strength $\left(\beta_{i}=\beta_{j}\right)$, then $\left.a_{i}^{*}\right|_{n=2}=1 / 2$ and $\left.a_{i}^{*}\right|_{n=3}=2 / 3$.

\section{THE MULTI-STAGE SETTING}

The model laid out in the foregoing leads to the strong conclusion that increasing the number of points awarded to a win, from $n=2$ to $n=3$, should increase the team's chosen degree of attacking efforts. The model is rather simple, of course, and the question arises as to how robust the predictions of the model are. An obvious limitation of the model is that it is a one-shot game- the two teams choose their actions once, at the beginning of the game. To be sure, such a static setting does capture some important strategic elements of a soccer match. Players are typically fairly specialized in their skills, and the characteristics of the starting eleven players (e.g., the number of defenders, midfielders and forwards that are fielded) does entail a degree of precommitment to the possible strategies that a team can pursue during the match. On the other hand, teams do retain some capacity to modify their degree of offensive play during the match. FIFA rules allow up to three of the starting players to be substituted at any point; bringing in a forward to replace a midfielder (or a defender), for example, is a common tactical adjustment for teams that are behind in the 
latter stages of a match. Even with the same set of players, some flexibility remains on how they are employed on the pitch to pursue a more (or less) aggressive strategy.

To model the situation in which teams can change their actions during the course of the match, as the "state" of the match evolves, requires consideration of at least two state variables: the current score of the match, and the time of play that remains. Rational contestants would be expected to adjust their chosen degree of offensive play according to whether they are winning, losing, or the current score is a tie. A team that is behind may have a stronger incentive to adopt a more attacking formation, and conversely, a team that is ahead may want to defend the lead. Furthermore, the relative incentive to attack or defend the current score may itself depend on how much time is left to play: when the end of the match is imminent, the option to wait to exert the maximum (and riskiest) attacking action would seem to be less and less desirable.

An attractive way to model this dynamic context might be to conceive of the match as made up of many stages, and to apply the structure developed earlier to each of the stages. The simplest version of that is to suppose, as in Brocas and Carrillo (2004), that the match is divided into two periods, and that at most one goal can be scored in each period. The two contestants choose actions $a_{i t} \in[0,1], i \in\{A, B\}$, in stage $t \in\{1,2\}$. The probability that team $i$ scores in stage $t$ is written as $p_{i}\left(a_{i t}, a_{j t}\right)$, where the functions $p_{i}(\cdot, \cdot)$ continue to satisfy the regularity conditions of Assumption 1 . In stage $t=1$, at the beginning of the match, the teams are tied, but in stage $t=2$ there are three different possible states of the game: team $A$ is ahead (it scored a goal in stage 1) while team B is behind, or team B is ahead (it scored in stage 1) while team A is behind, or the two teams are still tied (neither scored in stage 1). The payoff to each team in this game clearly depends on the entire action profile $\left(a_{A 1}, a_{A 2}, a_{B 1}, a_{B 2}\right)$. To rule out equilibrium profiles that rely on non-credible threats, the natural equilibrium concept in this setting, as discussed in Fudenberg and Tirole (1991), is that of Markov Perfect Nash Equilibrium (MPNE), that is, a subgame-perfect Nash equilibrium in Markov strategies, whereby only payoff-relevant state variables affect the agents' equilibrium action choices.

At the beginning of stage 2 , let the number of goals by which a team is behind (or ahead) denote the state of the game, so that from the perspective of team $i$ the state variable is $s_{i} \in\{-1,0,1\}$ (clearly, $s_{A}+s_{B}=0$ ), and denote with $U_{i 2}\left(a_{i 2}, a_{j 2} ; n, s_{i}\right)$ team $i$ 's payoff at 
stage 2 (this is the total payoff because the game ends with this stage). Then the payoff functions for the three possible states at $t=2$ are

$$
\begin{aligned}
& U_{i 2}\left(a_{i 2}, a_{j 2} ; n,-1\right)=p_{i}\left(a_{i 2}, a_{j 2}\right) \\
& U_{i 2}\left(a_{i 2}, a_{j 2} ; n, 0\right)=1+(n-1) p_{i}\left(a_{i 2}, a_{j 2}\right)-p_{j}\left(a_{j 2}, a_{i 2}\right) \\
& U_{i 2}\left(a_{i 2}, a_{j 2} ; n, 1\right)=n-p_{j}\left(a_{j 2}, a_{i 2}\right)(n-1)
\end{aligned}
$$

where $i, j \in\{A, B\}, j \neq i$. So when $s_{i}=0$, the $t=2$ subgame is essentially the same as that characterized earlier for the one-stage game. This subgame is supermodular and displays increasing differences, so that an increase in $n$ unambiguously increases the action levels chosen by the two teams. When a team is behind $\left(s_{i}=-1\right)$, the best a team can do is to tie by scoring a goal, and hence the payoff to the team is simply the probability of scoring (recall that a tie is worth one point). Because by Assumption 1 scoring probabilities are monotonically increasing in actions, team $i$ 's best action is found at the boundary of the action set, that is, $a_{i 2}^{*}=1$. Similarly, when a team is ahead, it does not gain by scoring in the last period, but it can lose $(n-1)$ points if the other team scores. So the team's best action is to minimize the probability of the other team scoring, which in view of the assumed monotonicity of probabilities requires the optimal state-contingent action $a_{i 2}^{*}=0$. Given these equilibrium actions at stage $t=2$, the equilibrium payoffs for the two teams at this stage are

$$
\begin{aligned}
& U_{i 2}^{*}(n,-1)=p_{i}(1,0) \\
& U_{i 2}^{*}(n, 0)=1+(n-1) p_{i}\left(a_{i 2}^{*}, a_{j 2}^{*}\right)-p_{j}\left(a_{j 2}^{*}, a_{i 2}^{*}\right) \\
& U_{i 2}^{*}(n, 1)=n-p_{j}(1,0)(n-1)
\end{aligned}
$$

where $i, j \in\{A, B\}, j \neq i$. The equilibrium at $t=1$ therefore hinges on the payoffs 


$$
\begin{aligned}
U_{i 1}\left(a_{i 1}, a_{j 1} ; n\right) & =U_{i 2}^{*}(n, 0)+\left[U_{i 2}^{*}(n, 1)-U_{i 2}^{*}(n, 0)\right] \cdot p_{i}\left(a_{i 1}, a_{j 1}\right) \\
& -\left[U_{i 2}^{*}(n, 0)-U_{i 2}^{*}(n,-1)\right] \cdot p_{j}\left(a_{j 1}, a_{i 1}\right), \quad, \quad i, j \in\{A, B\}, j \neq i .
\end{aligned}
$$

From the foregoing expressions it is verified that neither the supermodularity condition $\partial^{2} U_{i 1} / \partial a_{j 1} \partial a_{i 1} \geq 0$ nor the increasing difference condition $\partial^{2} U_{i 1}\left(a_{i 1}, a_{j 1} ; n\right) / \partial a_{i 1} \partial n \geq 0$ need hold, in general. Thus, one cannot conclude that increasing the number of points awarded to a win necessarily increases the teams' attacking choices at every stage and state of the game, which calls into question the observable implications of Proposition 2.

\section{A. Example II}

The parameterization in equation (2), when applied to the foregoing two-stage model, actually yields results that do accord with the predictions of Propositions 1 and 2. But consider instead the following parameterization presented in Brocas and Carrillo (2004):

$$
p_{i}=\kappa a_{i}+\ell a_{j}^{2} / 2
$$

where the parameters $\kappa$ and $\ell$ are strictly positive (ensuring monotonicity). Then it is readily verified that an increase in the number of points awarded to a win from $n=2$ to $n=3$ can in fact decrease the equilibrium action choices at stage $t=1$. As noted, for $s_{i}=-1$ and $s_{i}=1$ we have $a_{i 2}^{*}=1$ and $a_{i 2}^{*}=0$, respectively, regardless of the value of $n$. But for $s_{i}=0$ and for the case of an interior solution, $\left.a_{i 1}^{*}\right|_{n=2}=\left.a_{i 2}^{*}\right|_{n=2}=\kappa / \ell,\left.a_{i 2}^{*}\right|_{n=3}=2 \kappa / \ell$ and

$\left.a_{i 1}^{*}\right|_{n=3}=(\kappa / \ell)\left(2 \ell(1-\kappa)-4 \kappa^{2}\right) /\left(\ell(1-\kappa)+4 \kappa^{2}\right)$. Thus $\left.a_{i 1}^{*}\right|_{n=3}<\left.a_{i 1}^{*}\right|_{n=2}$ is certainly possible and will attain if $\ell(1-\kappa)<8 \kappa^{2}$. For example, for $\kappa=0.23$ and $\ell=0.5$ one finds $\left.a_{i 1}^{*}\right|_{n=2}=\left.a_{i 2}^{*}\right|_{n=2}=0.46,\left.a_{i 2}^{*}\right|_{n=3}=0.92$ and $\left.a_{i 1}^{*}\right|_{n=3}=0.43$.

Having shown that the result of Proposition 1 need not hold in each of the stages of a two-stage game, the question remains as to what that means for the overall outcome of the match. Brocas and Carillo (2004) conclude that the average degree of offensive play, that is, $\left(a_{i 1}^{*}+a_{i 2}^{*}\right) / 2$, could decrease as the number of points awarded to a win is increased, a case 
that would require $\ell<2 \kappa^{2} /(1-\kappa)$. But it turns out that this situation cannot arise when one accounts for the restrictions on the admissible parameter space. At a minimum one needs to ensure that the probability of a draw is nonnegative. Here, for states $s_{i} \neq 0$ the condition is $p_{i}(1,0)+p_{i}(0,1) \leq 1$, and for state $s_{i}=0$ the condition is $2 p_{i}\left(a_{i t}^{*}, a_{j t}^{*}\right) \leq 1$. The parametric restrictions associated with these conditions are $2 \kappa+\ell \leq 2$ and $\ell \geq 8 \kappa^{2}$, respectively (the latter applies for $n=3$ at $t=2)$, and in this parametric region it is clear that $\ell>2 \kappa^{2} /(1-\kappa)$. Alternatively, one might want to ensure that $p_{i}\left(a_{i}, a_{j}\right)+p_{j}\left(a_{j}, a_{i}\right) \leq 1$ holds for all $\left(a_{i}, a_{j}\right) \in[0,1] \times[0,1]$, which requires $2 \kappa+\ell \leq 1$, and also that an interior solution attains for both $n=2$ and $n=3$, which requires $2 \kappa \leq \ell$. The relevant parameter region in this case is $0<\kappa \leq 1 / 4$ and $2 \kappa \leq \ell \leq 1-2 \kappa$, again implying that the condition for the average degree of offensive play to decline cannot hold.

More to the point, we are concerned with the implications addressed by Proposition 2. The current example is of interest because one can show that, with the parameterization in (3), it is always the case that the expected number of goals are increased, and the probability of a draw is decreased, when the number of points awarded to a win increases from $n=2$ to $n=3$-regardless of whether or not the attacking actions of the teams increase in every stage of the game. That is, if $y^{*}$ denotes the expected number of goals and $z^{*}$ denotes the probability that the match ends in a draw when teams follow their equilibrium strategy, then $\left.y^{*}\right|_{n=3}>\left.y^{*}\right|_{n=2}$ and $\left.z^{*}\right|_{n=3}<\left.z^{*}\right|_{n=2}$. The details of the derivation are somewhat tedious and are sketched out in the Appendix. For example, for the specific instance of $\kappa=0.23$ and $\ell=0.5$, which does entail that first-stage actions decrease with $n=3$, as noted earlier, one finds $\left.y^{*}\right|_{n=2}=0.69,\left.y^{*}\right|_{n=3}=1.03,\left.z^{*}\right|_{n=2}=0.54$, and $\left.z^{*}\right|_{n=3}=0.18$.

\section{B. Computational Results}

Example II is intriguing. It illustrates the point made by Brocas and Carrillo (2004) that the one-stage model's prediction of Proposition 1-namely, that the teams' attacking actions uniformly increase as the number of points awarded to a win increases from $n=2$ to $n=3$ - need not extend to each stage of a multi-stage setting. Yet, as discussed, with this parametric specification the observable implications of the one-stage model do carry over to 
the two-stage setting. Specifically, the expected number of goals increases (Proposition 2(b)) and the probability of a draw decreases (Proposition 2(a)) as the number of points awarded to a win increases from $n=2$ to $n=3$. How general is this conclusion? Analytical results that apply generally appear difficult to establish in our context, in view of the fact that the supermodularity condition $\partial^{2} U_{i 1} / \partial a_{j 1} \partial a_{i 1} \geq 0$ and/or the increasing difference condition $\partial^{2} U_{i 1} / \partial a_{i 1} \partial n \geq 0$ may not hold in stage $t=1$. Thus, I investigate the problem at hand with an extensive experiment that computes the MPNE given a specific_-and fairly generalparameterization of the probability function evaluated over a large set of possible parameter values.

The specific parameterization that I use is

$$
p_{i}=\gamma\left[\left(1+\beta_{i}-\beta_{j}\right)\left(a_{i}-\lambda a_{i}^{2} / 2\right)+\phi a_{j}^{2} / 2+\eta a_{i} a_{j}\right] \quad i, j \in\{A, B\}, j \neq i
$$

where $\gamma>0$ is a scaling parameter that ensures that actions are mapped into the admissible probability space $[0,1] \times[0,1], \lambda \geq 0$ ensures that the probabilities are concave in own action, and $\phi \geq 0$ and $\eta \geq 0$ ensure that probabilities are increasing in the other team's action. The skill parameters $\beta_{i}, \beta_{j} \geq 0$ matter only in their difference $\beta_{i}-\beta_{j}$, which can be of either sign. But for probabilities to be increasing in own action we need to restrict attention to $1+\beta_{i}-\beta_{j}>0$. Also, monotonicity for all $a_{i} \in[0,1]$ requires $\lambda \leq 1$. Note that the probability function in equation (4) encompasses the functions used in the two preceding examples as special cases. Specifically, (4) reduces to (2) for $\lambda=1, \quad \eta=0$, and $\phi=1$; and (4) is isomorphic to (3) for $\lambda=0, \quad \eta=0$ and $\beta_{i}=\beta_{j}$.

Further parametric restrictions are required to ensure that feasible actions do not yield a negative draw probability, and to restrict attention to the parametric domain that yields an interior solution when the state of the match is that of a draw. ${ }^{4}$ Recalling the expression for $U_{i 2}\left(a_{i 2}, a_{j 2} ; n, 0\right)$ given earlier, it is verified that for the probability function

\footnotetext{
${ }^{4}$ This is not a consistency requirement but is meant to restrict attention to the domain of interest. Clearly, if a corner solution $a_{i}=1$ were to attain when the teams are tied, implying that the teams are already adopting the highest possible attacking choices, there would be little scope for the 3-point system.
} 
in equation (4), $\partial U_{i 2} / \partial a_{i 2}=(n-1) \gamma\left[\left(1+\beta_{i}-\beta_{j}\right)\right]$ when evaluated at $a_{i 2}=a_{j 2}=0$, and thus the assumed monotonicity condition $1+\beta_{i}-\beta_{j}>0$ guarantees strictly positive equilibrium action choices. Similarly, when evaluated at $a_{i 2}=a_{j 2}=1$ we have $\partial U_{i 2} / \partial a_{i 2}=\gamma\{(n-1)[(1+\beta)(1-\lambda)]-\phi+\eta(n-2)\}$. This expression is increasing in $n$, so the restrictive case for us is $n=3$. For the expression to be non-positive at $n=3$ (entailing interior best responses) we therefore need $2[(1+\beta)(1-\lambda)]+\eta \leq \phi$. Finally, because $p_{A}+p_{B}=\gamma[2-\lambda+\phi+2 \eta]$ when evaluated at $a_{i 2}=a_{j 2}=1$, for a non-negative draw probability we need $\gamma[2-\lambda+\phi+2 \eta] \leq 1$.

The two-stage model with the probability specification of equation (4) involves five parameters: $\gamma, \lambda, \phi, \eta$ and $\beta \equiv \beta_{i}-\beta_{j}$. For each of these parameters I considered 11 distinct values, equally spaced on the following supports: $\gamma \in[0.1,0.5], \beta \in[0,0.5], \lambda \in[0,1]$, $\phi \in[0,3]$ and $\eta \in[0,3]$. This gives $11^{5}=161,051$ possible parameter combinations. Of these, I drop the 1,331 parameter combinations for which we have both $\phi=0$ and $\eta=0$ (implying violation of Assumption 1(b), i.e., no strategic interaction). For the set of parameters that remains, I find that 12,264 parameter combinations satisfy the regularity restrictions discussed in the foregoing. For each of these 12, 264 parameter vectors, I solved for the MPNE of the two-stage game, by using a user-written program coded in MATLAB 7, for both the cases of $n=2$ and $n=3 .^{5}$ In addition to the teams' equilibrium actions, for each instance I computed the expected number of goals and the probability that the match would end in a draw (the form of these functions for this two-stage setting is given in the Appendix). I find that of the 12,264 games solved, in 120 cases the first-stage action choice of at least one team is negatively affected, i.e., $\left.a_{i 1}^{*}\right|_{n=3}<\left.a_{i 1}^{*}\right|_{n=2}$. Thus, the one-stage model prediction that the teams' attacking choices are increased by an increase in the number of points awarded to a win does not extend to all possible situations that arise in the two-stage game (although it does apply to $99 \%$ of the cases examined). But, in all 12,264 games solved, I find that the expected number of goals is never lower with $n=3$ than with $n=2$,

\footnotetext{
${ }^{5}$ The particular algorithm that I implement iterates the maximization of the Nikaido-Isoda function (Uryasev and Rubinstein, 1994).
} 
and similarly the probability of a draw never increases when the number of points awarded to a win increases from $n=2$ to $n=3$.

In conclusion, it seems that the observable implications predicted by the static model - specifically the results in Proposition 2-are rather robust and do extend to the multi-stage setting for a fairly general parametric specification of the model that has been postulated. Thus, I propose to empirically test the implications of Proposition 2(a) and Proposition 2(b).

\section{EMPIRICAL RESULTS}

To test the empirically observable predictions of the model, a large data set was collected on recorded score outcomes before and after the implementation of the 3-point rule. The data were assembled by compiling information reported by the Rec.Sport.Soccer Statistics Foundation (RSSSF). This organization, dedicated to collecting a variety of statistics for soccer competitions from around the world, has built a substantial data archive that is accessible online. ${ }^{6}$ From the Foundation's country-specific annual final tables for the top national competitions, a dataset was constructed which includes 35 countries over the last 30 years. One pre-requisite for a country's inclusion in this study was the availability of a consistent time series of national competition outcomes that spans both the 2-point system and the 3-point system. In the end, the sample does include virtually all of the major soccerpracticing countries, as well as providing a broad geographic representation. The set of countries considered, along with the date of their adoption of the 3-point system, is reported in Table 1. The choice of the last 30 years ensures that the sample includes comparable information for the 2-point and the 3-point systems (specifically, in the final sample, about $48 \%$ of the observations pertain to the 3-point system).

For each country and each year, the data for the annual national competition were used to compute two summary statistics that, in view of Proposition 2, are of direct interest: the per-match average number of goals, and the proportion of games ending in a draw. For each country and each year, I also recorded the number of teams taking part in the competition, as well as the year in which the country first adopted the 3-point rule. A brief description of the data is provided in Table 2. For some countries in some years, the

\footnotetext{
${ }^{6}$ As of August 2007, the working URL for the Foundation's Web site was http://www.rsssf.com.
} 
tournament organization did not have the usual round-robin structure. ${ }^{7}$ A common feature in such cases was an initial phase with round-robin organization, with qualifying teams playing a final playoff phase. In those instances, therefore, the average number of goals per match and fraction of drawn matches that I computed for that year and that country relied only on the round-robin portion of the tournament. I note that 30 years and 35 countries give us potentially 1,050 observations. But some observations had to be dropped because in that year and country the point system in place was neither the 2-1-0 nor the 3-1-0 system. Also, a few national competitions for the last year of the sample (2007) had not yet been completed when this study was executed. In the end, therefore, the sample encompasses 1,028 observations.

\section{A. Expected Number of Goals}

The data at hand is a classic panel data set and lends itself to a straightforward procedure to test the hypotheses of interest. To analyze the prediction of Proposition 2(b), that the expected number of goals ought to increase under the 3-point system, the most general model that I consider is written as

$$
y_{i t}=X_{i t} \alpha+\delta D_{i t}+\theta_{i}+e_{i t}
$$

where $y_{i t}$ is the average number of goals observed in country $i(i=1,2, \ldots, 35)$ in year $t(t=1978, \ldots, 2007), D_{i t}$ is a dummy variable that takes value 1 if the 3 -point system is in place in country $i$ at time $t$ and value 0 otherwise, $X_{i t}$ is a vector of conditioning variables (including an intercept) that apply for country $i$ at time $t, \theta_{i}$ is a term that captures country-specific effects, $e_{i t}$ is a zero-mean error term that is presumed identically and independently distributed across observations, and $(\alpha, \delta)$ are parameters to be estimated. The parameter $\delta$ directly relates to the hypothesis of interest: rejection of the null hypothesis $H_{0}: \delta=0$ (against the alternative $H_{1}: \delta>0$ ) would provide support for the predictions of Proposition 2.

\footnotetext{
${ }^{7}$ For example, only starting in 2003 did Brazil adopt the double round-robin structure that is the norm in most other countries.
} 
As is standard in the estimation of error-component models, the country-specific effects $\theta_{i}$ can be regarded as either fixed or random (Wooldridge, 2002). Here only the results of the fixed-effects model, which is essentially unaffected by the slight unbalanced nature of the data, are reported. But I note that the random-effects model yields essentially identical results. In fact, in all cases the fixed and random effect models are statistically indistinguishable according to the standard Hausman test (1978). Estimation results for the model in equation (5) are reported in Table $3 .^{8}$ Four versions of the model are considered. In all models the country-specific fixed effects turn out to be highly significant. ${ }^{9}$ In model I, only a constant for the intercept is included in the conditioning vector $X_{i t}$, so that only the 3-point dummy variable is included in the explanatory variables. The t-ratio of the estimated parameter $\hat{\delta}$ of the 3-point dummy variable is 9.63, which would indicate a decisive rejection of the null hypothesis $H_{0}: \delta=0$. Hence, the evidence from this model is that the panel data set is consistent with a significant effect of the 3-point rule in the expected direction.

To investigate the robustness of this conclusion, the model was estimated with some additional conditioning variables. In model II, a linear time trend is introduced in the conditioning vector. Because the 3-point dummy variable is correlated with time the simple correlation coefficient between the two variables in the sample is 0.81), the general issue arises concerning the possibility that the variable $D_{i t}$ might be picking up a secular trend on the average number of goals. In fact, such a secular trend clearly is a property of the data prior to the introduction of the 3-point system, and, indeed, concern about such a trend was arguably a motivation for FIFA to introduce the 3-point system in the mid-1990s. To illustrate that trend, for a few leading soccer countries I collected a more extensive dataset that covers the entire post-WWII period prior to the introduction of the 3-point system. Figure 1 reports the average number of goals and the fractions of drawn matches for four leading European soccer countries (France, Germany, Italy and Spain), and Figure 2 reports the same statistics for three leading South-American soccer countries (Argentina, Chile and

\footnotetext{
${ }^{8}$ Estimation was carried out in TSP 5.0.

9 For example, for model I the F test for a common intercept is $F(34,992)=32.23$, which exceeds the appropriate critical value at virtually any significance level.
} 
Uruguay). ${ }^{10} \mathrm{~A}$ secular trend toward a decline in the average number of goals per match, as well as an increase in the fraction of games ending in a draw, is clearly present. A number of reasons might explain this trend, including the increased physical conditioning and athletic prowess of professional soccer players (which, coupled with an essentially unchanged soccer pitch size, might have increased the effectiveness of soccer defense). Here I are not concerned with explaining the dynamics of these trends; rather, I wish to account for such a background element in assessing the significance of the 3-point rule effects, and that is accomplished by introducing a linear trend among the conditioning variables.

The introduction of a linear trend among the conditioning variables does change the size of the parameter of interest, $\hat{\delta}$, but leaves its statistical significance level essentially unaltered (the t-ratio is now 8.18). The estimated coefficient of the trend variable is itself negative, consistent with the existing underlying secular trend prior to the introduction of the 3-point system illustrated in Figures 1 and 2.

Another conditioning variable that was considered in models III and IV is the number of teams taking part in the round-robin tournament for which the average statistics used as observations were computed. This number turned out to vary both across countries and, to some extent, has also varied over time within individual countries (Table 2). ${ }^{11}$ Although the effect of tournament-group size was not explicitly considered in the theoretical model, it seems likely that the marginal strategic incentive of the 3-point system is reduced when fewer teams are involved. (In the limiting case of only two teams, only victory matters, and the number of points awarded to it are meaningless.) Another reason that the size of the tournament group matters has to do with heterogeneity of teams. As suggested in the theoretical model discussed earlier, increased differences across teams increase the expected number of goals, ceteris paribus. The presumption here would be that championships with many teams may be more heterogeneous than championships with fewer teams (although,

\footnotetext{
${ }^{10}$ Data for the other major European soccer country, England, are not used in Figure 1 because England introduced the 3-point system much earlier (in the 1981/82 season) than the four countries used here (see Table 1). Data for the other major South-American soccer country, Brazil, are not used in Figure 2 because of the difficulty of assembling a consistent time series for the entire period of interest here.

${ }^{11}$ Indeed, the smallest (5) and largest (28) group sizes in the sample were both observed in Brazil (in years 1982-84 and 2001, respectively).
} 
admittedly, the number of teams might be a weak proxy for that effect). Consistent with the foregoing discussion, the estimated parameter of this variable turns out to be positive and statistically significant at the 0.01 probability level in both models III and IV. In any event, inference concerning the parameter of interest is not affected: the hypothesis $H_{0}: \delta=0$ is consistently rejected, at the 0.01 probability level, across all models.

The conclusion of this econometric analysis, therefore, is that the 3-point system did make a difference, and the added incentive has had a statistically significant impact on the expected number of goals. This evidence is quite consistent with the strategic model that was postulated and provides support for some basic implications of Nash equilibrium in a strategic setting. Although the statistical significance of the effect is strong, the quantitative

impact of the 3-point rule on goals is (perhaps not surprisingly) somewhat limited. Recalling that the overall average number of goals per match in the sample is approximately 2.62 , the predicted increase in the expected number of goals per match implied by the results of Table 3 ranges from $5.7 \%$ (model I) to $8.5 \%$ (model IV).

\section{B. Probability of Drawn Matches}

As noted earlier, in addition to computing the average number of goals for each country in each year, the fraction of games that ended in a draw for each of the sample points was also computed. Proposition 2(a) can then be tested directly. The analysis proceeds along the same lines as for the expected number of goals. The estimated model is

$$
z_{i t}=X_{i t} \omega+\rho D_{i t}+\mu_{i}+v_{i t}
$$

where $z_{i t}$ is the fractions of matches that ended in a draw observed in country $i$ $(i=1,2, \ldots, 35)$ in year $t(t=1978, \ldots, 2007), D_{i t}$ and $X_{i t}$ are as defined earlier, $\mu_{i}$ is the term that captures country-specific effects, $v_{i t}$ denotes the zero-mean i.i.d. error term, and $(\omega, \rho)$ label the parameters to be estimated. The null hypothesis of interest is $H_{0}: \rho=0$, rejection of which (against the alternative $H_{1}: \rho<0$ ) would provide support for the predictions of Proposition 2(a). As for the case of the expected number of goals, I report the fixed-effect estimation results for four models that differ in the set of conditioning variables. 
The estimation results for model (6), summarized in Table 4, are quite consistent with those of model (5). The introduction of the 3-point system appears to have had a statistically significant impact on the expected number of draws in the direction predicted by Proposition 2(a). In particular, the null hypothesis $H_{0}: \rho=0$ is rejected at the 0.01 probability level in all versions of the model. The other conditioning variables also have a sign consistent with the effects of Table 3. In particular, allowing for the effect of a time trend has a strong impact on the size of the 3-point effect. Recalling that the overall average of matches ending in a draw in the sample is approximately 0.27 , the quantitative impact of the introduction of the 3-point rule implied by the results of Table 4 is a decrease in the fraction of drawn matches that ranges from $8.8 \%$ (model I) to $16.2 \%$ (model IV). The impact of the 3-point rule on decreasing the number of matches that end in a draw thus appears quantitatively higher than the impact on increasing the expected number of goals.

\section{Country-specific Responses}

The results reported in Tables 2 and 3 allow for country-specific fixed effects and maintain a common response to the structural change of the 3-point system. In view of the variety of styles that different countries bring to the practice of soccer, exploring the implications of relaxing this last restriction is of some interest. Thus, the models in equations (5) and (6) were re-estimated by allowing for country-specific responses to the 3-point dummy variable (i.e., by allowing the parameters $\delta$ and $\rho$ to vary by country). Table 5 reports the most general version of the models in question, which includes trend and number of teams as conditioning variables. Perhaps not surprisingly, we find considerable variability in these country-specific responses. For the model explaining the expected number of goals, we find 27 individual responses that are statistically significant at at least the 10\% level. Of these, 24 have the expected positive sign, consistent with the average response of Table 3. NonEuropean countries display a consistently positive impact of the 3-point system. European countries tend to display a smaller impact, and in a few cases the country-specific effect is actually negative. In particular, for Austria, Germany and the Czech Republic the estimated impact is negative and statistically significant.

For the model explaining the proportion of matches that end in a draw, also reported in Table 5, we find that all but 2 of the estimated country-specific responses have the expected negative sign (and 27 of these are statistically significant at at least the $10 \%$ level). 
Of the two responses with a positive sign, one (for Italy) also appears statistically significant. Overall, however, the more disaggregated results of Table 5 are quite consistent with the average results reported in Tables 2 and 3. Of course, the game of soccer is much more complex than the simple representation provided by the model of this article, and considerable variability in outcomes remains to be explained. But the evidence that I have presented nonetheless allows us to conclude that the predictions of Propositions 2 find considerable support in the data. The 3-points-for-a-win system does appear to have had a statistically significant impact on observed outcomes, although its magnitude is somewhat limited and displays considerable variability across countries.

\section{CONCLUSION}

In this article I have investigated the impact of a major structural change in the organization of soccer competitions that took place in the mid-1990s, specifically the introduction of the 3-points-for-a-win system. I start by providing a simple game-theoretic representation of the relevant strategic setting that is amenable to strong comparative statics predictions. Based on both analytical results and numerical computation, I conjecture that the "natural experiment" of the 3-points-for-a-win change should have increased the expected number of goals per match and decreased the fraction of drawn matches. Such predictions are then evaluated empirically by means of a large and original data set that was assembled. By using a panel data analysis that spans 35 countries and 30 years, we find that the results are quite supportive of the game-theoretic predictions. Specifically, there is a statistically significant effect of the 3-points-for-a-win system on both goals scored and expected draws. The magnitude of these effects, while not large in an absolute sense, is not inconsequential. The most general model with common (across all countries) response to the 3-point system yields an estimated increase in number of goals per match of $8.5 \%$ and an estimated decline in the probability of drawn matches of $16.2 \%$.

The results of this article should be of interest from several perspectives. First, the analysis adds to a small number of existing studies that lend empirical support to the notion of Nash equilibrium. Specifically, I show that a game-theoretic approach to modeling strategic interactions can provide sharp predictions that are consistent with observed behavior. The results are also of interest to the organization of sport competitions. As many other studies have shown in other and related settings, incentives do matter, and, given a 
well defined objective function, it may be possible to improve the entertainment value of sporting events by tailoring the rules of the game to a specific desired objective (more goals, fewer ties!).

\section{REFERENCES}

Brocas, I., and J.D. Carrillo. "Do the Three-Point Victory and Golden Goal Rules Make Soccer More Exciting?” Journal of Sports Economics, 5(2), 2004, 169-185.

Cabral, L.M.B. “R\&D Competition when Firms Choose Variance.” Journal of Economics \& Management Strategy, 12, 2003, 139-150.

Chiappori, P.A., S. Levitt, and T. Groseclose. "Testing Mixed Strategy Equilibria When Players are Heterogeneous: The Case of Penalty Kicks in Soccer." American Economic Review, 92(4), 2002, 1138-1151.

Correira Guedes, J., and F.S. Machado. "Changing Rewards in Contest: Has the Three-Point Rule Brought More Offense to Soccer?” Empirical Economics, 27, 2002, 607-630.

Dasgupta, P., and E. Maskin. "The Simple Economics of Research Portfolios.” Economic Journal, 97, 1987, 581-595.

Ferrall, C., and A.A. Smith, Jr. "A Sequential Game Model of Sports Championship Series: Theory and Estimation.” Review of Economic and Statistics, 81(4), 1999, 705-719.

Frick, B. "Contest Theory and Sport." Oxford Review of Economic Policy, 19(4), 2003, 512529.

Fudenberg, D., and J. Tirole. Game Theory. Cambridge, MA: The MIT Press, 1991.

Garicano, L., and I. Palacios-Huerta. "Sabotage in Tournaments: Making the Beautiful Game a Bit Less Beautiful.” CEPR Discussion Paper No. 5231, September 2005.

Hausman, J.A. "Specification Tests in Econometrics." Econometrica, 46, 1978, 1251-1271. Hsu, S-H., C-Y. Huang, and C-T. Tang, "Minimax Play at Wimbledon: Comment." American Economic Review, 97(1), 2007, 517-523.

Lazear, E., and S. Rosen. "Rank-Order Tournaments as Optimum Labor Contracts." Journal of Political Economy, 89(5), 1981, 841-866.

Moschini, G. "Nash Equilibrium in Strictly Competitive Games: Live Play in Soccer." Economics Letters, 85(3), December 2004, 365-371.

Noll, R.G. "The Organization of Sport Leagues." Oxford Review of Economic Policy, 19(4), 2003, 530-551. 
Palacios-Huerta, I. "Professionals Play Minimax." Review of Economics Studies 70(2), 2003, $395-415$.

Szymanski, S. "The Economic Design of Sport Contests.” Journal of Economic Literature, 41, December 2003, 1137-1187.

Topkis, D. "Equilibrium Point in Nonzero-Sum n-Person Submodular Games." SIAM Journal on Control and Optimization, 17, 1979, 773-787.

Uryasev, S., and , R.Y. Rubinstein. "On Relaxation Algorithms in Computation of Noncooperative Equilibria." IEEE Transactions on Automatic Control, 39(6), 1994, 1263-1267.

Vives, X. "Nash Equilibrium with Strategic Complementarities." Journal of Mathematical Economics, 19, 1990, 305-321.

Vives, X. "Complementarities and Games: New Developments.” Journal of Economic Literature, 43, 2005, 437-479.

Walker, M., and J. Wooders. "Minimax Play at Wimbledon." American Economic Review, 91(5), 2001, 1521-1538.

Wooldridge, J.M. Econometric Analysis of Cross Section and Panel Data. Cambridge, MA: The MIT Press, 2002. 
TABLE 1. Introduction of the 3-points-for-a-win system

\begin{tabular}{|c|c|}
\hline Year* & Countries in the sample \\
\hline 1982 & England \\
\hline 1983 & Israel \\
\hline 1988 & Norway and Turkey \\
\hline 1989 & Hungary, Sweden \\
\hline 1993 & Australia, Greece \\
\hline 1994 & Bulgaria, Ireland \\
\hline 1995 & $\begin{array}{l}\text { Brazil, Chile, Colombia, Czech Rep., Egypt, France, Italy, Romania, } \\
\text { Russia, }{ }^{\text {, S Scotland, Uruguay }}\end{array}$ \\
\hline 1996 & $\begin{array}{l}\text { Argentina, Austria, Belgium, Denmark, Germany, }{ }^{\dagger} \text { Mexico, Netherlands, } \\
\text { Poland, Portugal, Serbia, }{ }^{\dagger} \text { Spain, Switzerland, Tunisia }\end{array}$ \\
\hline 1997 & Albania \\
\hline
\end{tabular}

Notes: * Year refers to the end of the season when the season spans two calendar years (e.g., 1982 means the 1981/82 season for England).

${ }^{\#}$ Czechoslovakia up to $1993 .{ }^{\S}$ Soviet Union up to $1991 .{ }^{\dagger}$ Bundesliga (West Germany only prior to 1992). ${ }^{\ddagger}$ Yugoslavia prior to 1992.

TABLE 2. Description of the panel data set, 35 countries, 1978-2007

\begin{tabular}{lcccc}
\hline & mean & s.d. & min & max \\
\hline $\begin{array}{l}\text { Season's average number of } \\
\text { goals per match }\end{array}$ & 2.62 & 0.35 & 1.59 & 3.83 \\
$\begin{array}{l}\text { Fraction of matches that end } \\
\text { in a draw }\end{array}$ & 0.27 & 0.05 & 0.08 & 0.43 \\
$\begin{array}{l}\text { Number of teams in round- } \\
\text { robin tournament }\end{array}$ & 16.0 & 3.18 & 5 & 28 \\
\hline
\end{tabular}

Source: Computed from RSSSF archive data. 
TABLE 3. Estimation results: expected number of goals

\begin{tabular}{lcccc}
\hline Model & $\begin{array}{c}\text { 3-point } \\
\text { Dummy }\end{array}$ & Trend & $\begin{array}{c}\text { Number } \\
\text { of teams }\end{array}$ & $R^{2}$ \\
\hline I & $\begin{array}{c}0.14800 \\
(0.01536)\end{array}$ & & & 0.546 \\
& & & & \\
II & 0.23033 & -0.00550 & & 0.552 \\
& $(0.02815)$ & $(0.00158)$ & & \\
III & 0.14580 & & 0.01745 & 0.556 \\
& $(0.01522)$ & & $(0.00380)$ & \\
IV & 0.22330 & -0.00517 & 0.01686 & 0.560 \\
& $(0.02793)$ & $(0.00157)$ & $(0.00379)$ & \\
\hline
\end{tabular}

Standard errors are reported in parentheses.

Number of observations $=1,028$.

TABLE 4. Estimation results: fraction of games ending in a draw

\begin{tabular}{lcccc}
\hline Model & $\begin{array}{c}\text { 3-point } \\
\text { dummy }\end{array}$ & Trend & $\begin{array}{c}\text { Number } \\
\text { of teams }\end{array}$ & $R^{2}$ \\
\hline I & $\begin{array}{l}-0.02402 \\
(0.00263)\end{array}$ & & & 0.446 \\
& & & & \\
II & -0.04376 & 0.00132 & & 0.459 \\
& $(0.00480)$ & $(0.00027)$ & & \\
III & -0.02390 & & -0.00092 & 0.447 \\
& $(0.00263)$ & & $(0.00066)$ & \\
IV & -0.04343 & 0.00130 & -0.00077 & 0.459 \\
& $(0.00480)$ & $(0.00027)$ & $(0.00065)$ & \\
\hline
\end{tabular}

Standard errors are reported in parentheses.

Number of observations $=1,028$. 
TABLE 5. Country-specific response to 3-point dummy

\begin{tabular}{|c|c|c|c|c|}
\hline & \multicolumn{2}{|c|}{ Expected Number of Goals } & \multicolumn{2}{|c|}{ Fractions of Draws } \\
\hline & estimate & s.e. p-value & estimate & $\mathrm{p}$-value \\
\hline Trend & -0.0055 & $0.0014[.000]$ & 0.0013 & $0.0002[.000]$ \\
\hline $\mathrm{N}$ of teams & 0.0020 & $0.0043[.637]$ & -0.0006 & $0.0008[.398]$ \\
\hline \multicolumn{5}{|l|}{ 3-point dummy } \\
\hline Albania & 0.4559 & $0.0820[.000]$ & -0.1315 & $0.0146[.000]$ \\
\hline Argentina & 0.3865 & $0.0807[.000]$ & -0.0722 & $0.0143[.000]$ \\
\hline Australia & 0.3216 & $0.0815[.000]$ & -0.0623 & $0.0145[.000]$ \\
\hline Austria & -0.1885 & $0.0800[.019]$ & -0.0281 & $0.0142[.048]$ \\
\hline Belgium & 0.2904 & $0.0798[.000]$ & -0.0127 & $0.0142[.371]$ \\
\hline Brazil & 0.5471 & $0.1006[.000]$ & -0.0578 & $0.0178[.001]$ \\
\hline Bulgaria & 0.2168 & $0.0823[.009]$ & -0.0793 & $0.0146[.000]$ \\
\hline Chile & 0.4670 & $0.0790[.000]$ & -0.0736 & $0.0140[.000]$ \\
\hline Colombia & 0.1364 & $0.0808[.092]$ & -0.0300 & $0.0143[.037]$ \\
\hline Czech Rep. ${ }^{\#}$ & -0.1982 & $0.0790[.012]$ & 0.0224 & $0.0140[.110]$ \\
\hline Denmark & 0.2159 & $0.0801[.007]$ & -0.0353 & $0.0142[.013]$ \\
\hline Egypt & 0.5851 & $0.0803[.000]$ & -0.0892 & $0.0142[.000]$ \\
\hline England & 0.0823 & $0.1131[.467]$ & -0.0410 & $0.0201[.041]$ \\
\hline France & -0.0815 & $0.0790[.302]$ & -0.0193 & $0.0140[.168]$ \\
\hline Germany $^{\dagger}$ & -0.1627 & $0.0798[.042]$ & -0.0244 & $0.0142[.086]$ \\
\hline Greece & 0.3585 & $0.0783[.000]$ & -0.0609 & $0.0139[.000]$ \\
\hline Hungary & -0.0032 & $0.0812[.968]$ & -0.0581 & $0.0144[.000]$ \\
\hline Ireland & -0.1221 & $0.0809[.132]$ & -0.0118 & $0.0144[.410]$ \\
\hline Israel & 0.5218 & $0.1036[.000]$ & 0.0207 & $0.0184[.260]$ \\
\hline Italy & 0.5517 & $0.0794[.000]$ & 0.0676 & $0.0141[.000]$ \\
\hline Mexico & 0.2882 & $0.0800[.000]$ & -0.0574 & $0.0142[.000]$ \\
\hline Netherlands & 0.0648 & $0.0798[.417]$ & -0.0587 & $0.0142[.000]$ \\
\hline Norway & 0.4032 & $0.0865[.000]$ & -0.0564 & $0.0153[.000]$ \\
\hline Poland & 0.3467 & $0.0798[.000]$ & -0.0821 & $0.0142[.000]$ \\
\hline Portugal & 0.1876 & $0.0799[.019]$ & -0.0271 & $0.0142[.056]$ \\
\hline Romania & 0.0230 & $0.0790[.771]$ & -0.0124 & $0.0140[.376]$ \\
\hline Russia ${ }^{\Omega}$ & 0.1842 & $0.0805[.022]$ & -0.0353 & $0.0143[.014]$ \\
\hline Scotland & 0.1616 & $0.0790[.041]$ & -0.0317 & $0.0140[.024]$ \\
\hline Serbia ${ }^{\ddagger}$ & 0.0619 & $0.0845[.464]$ & -0.0800 & $0.0150[.000]$ \\
\hline Spain & 0.2167 & $0.0801[.007]$ & -0.0152 & $0.0142[.287]$ \\
\hline Sweden & 0.1674 & $0.0817[.041]$ & -0.0385 & $0.0145[.008]$ \\
\hline Switzerland & 0.0577 & $0.0803[.472]$ & -0.0345 & $0.0142[.016]$ \\
\hline Tunisia & 0.1596 & $0.0803[.047]$ & -0.0513 & $0.0142[.000]$ \\
\hline Turkey & 0.8950 & $0.0828[.000]$ & -0.1056 & $0.0147[.000]$ \\
\hline Uruguay & 0.5526 & $0.0799[.000]$ & -0.0687 & $0.0142[.000]$ \\
\hline$R^{2}$ & 0.672 & & 0.570 & \\
\hline
\end{tabular}

Notes: ${ }^{\#}$ Czechoslovakia up to $1993 .{ }^{\S}$ Soviet Union up to $1991 .{ }^{\dagger}$ Bundesliga (West Germany only prior to 1992). " Yugoslavia prior to 1992. 
FIGURE 1. Goals per match and fraction of drawn matches prior to 3-point system: France, Germany, Italy and Spain, 1946/47 to 1993/94

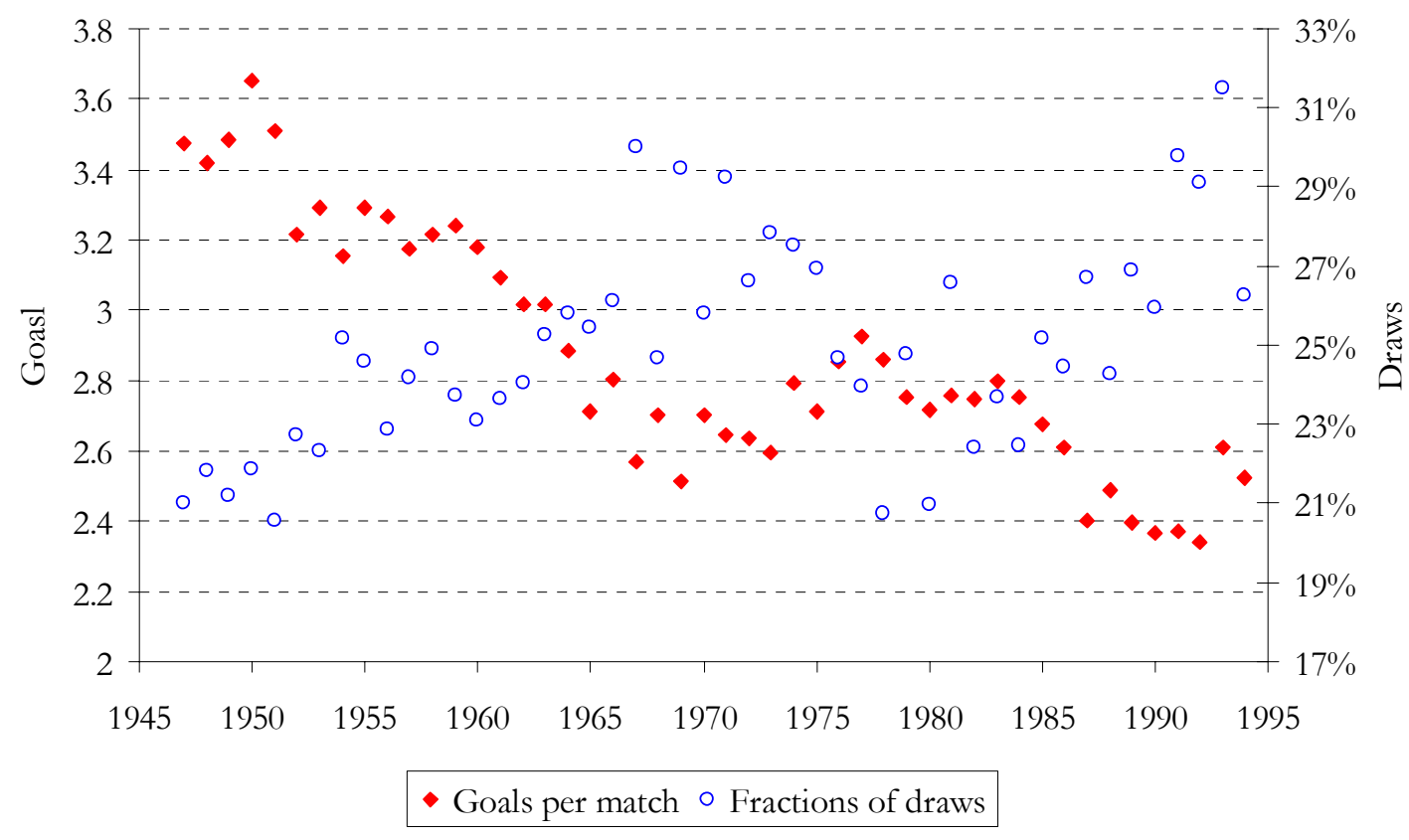

FIGURE 2. Goals per match and fraction of drawn matches prior to 3-point system: Argentina, Chile and Uruguay, 1947 to 1994

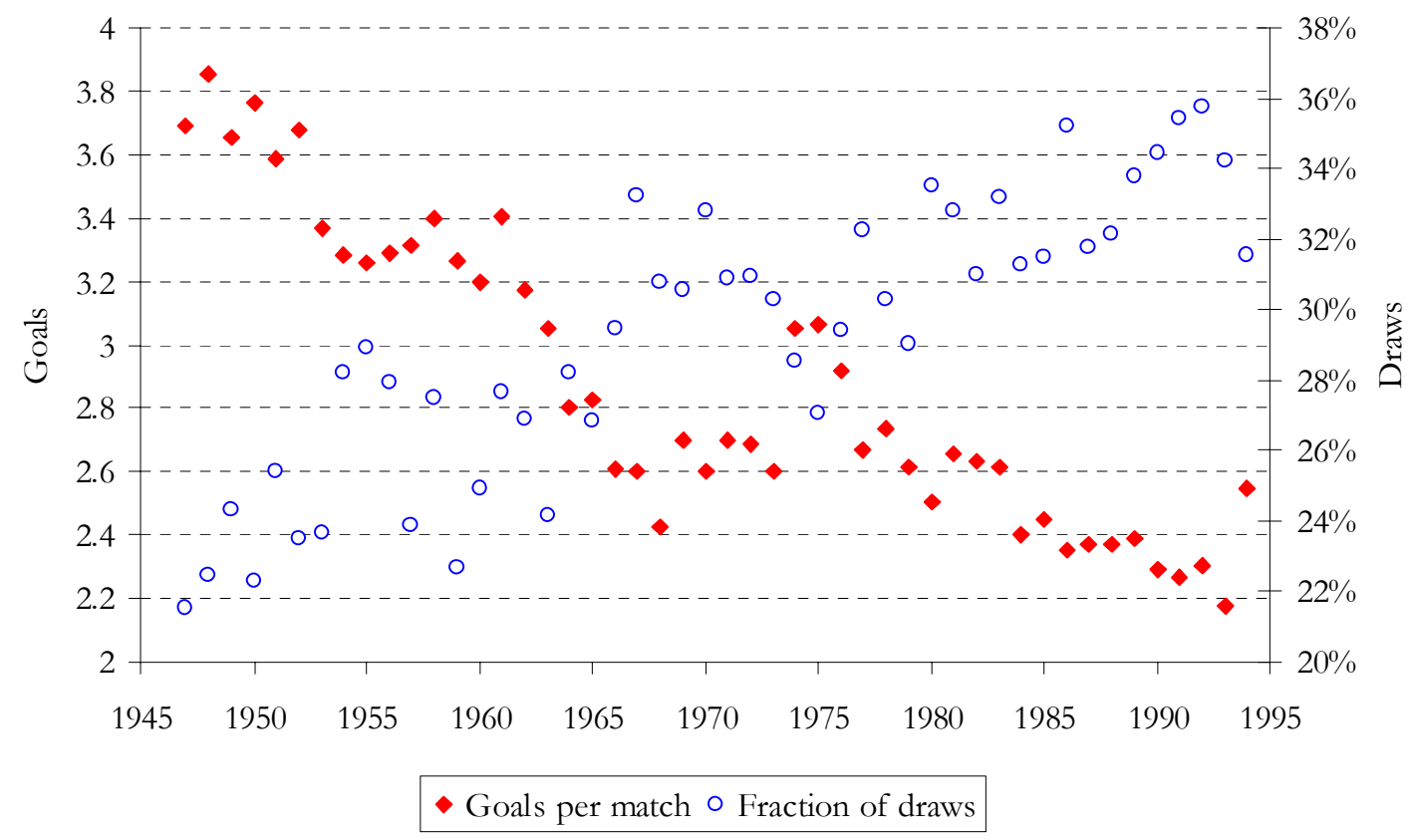




\section{APPENDIX}

For the general two-stage soccer model developed in the text, the expected number of goals when teams follow their equilibrium strategy is

$$
\begin{aligned}
y^{*}= & p_{i}\left(a_{i 1}^{*}, a_{j 1}^{*}\right)\left(1+p_{i}(0,1)+p_{j}(1,0)\right)+p_{j}\left(a_{j 1}^{*}, a_{i 1}^{*}\right)\left(1+p_{i}(1,0)+p_{j}(0,1)\right) \\
& +\left(1-p_{i}\left(a_{i 1}^{*}, a_{j 1}^{*}\right)-p_{j}\left(a_{j 1}^{*}, a_{i 1}^{*}\right)\right)\left[p_{i}\left(a_{i 2}^{*}, a_{j 2}^{*}\right)+p_{j}\left(a_{j 2}^{*}, a_{i 2}^{*}\right)\right] .
\end{aligned}
$$

As for a match ending in a draw, this event can arise because both teams fail to score in both stages, or because the team that is behind in the second stage scores. Thus, the probability that the match ends in a draw when teams follow their equilibrium strategy is

$$
\begin{aligned}
z^{*}= & \left(1-p_{i}\left(a_{i 1}^{*}, a_{j 1}^{*}\right)-p_{j}\left(a_{j 1}^{*}, a_{i 1}^{*}\right)\right)\left(1-p_{i}\left(a_{i 2}^{*}, a_{j 2}^{*}\right)-p_{j}\left(a_{j 2}^{*}, a_{i 2}^{*}\right)\right) \\
& +p_{i}\left(a_{i 1}^{*}, a_{j 1}^{*}\right) p_{j}(1,0)+p_{j}\left(a_{j 1}^{*}, a_{i 1}^{*}\right) p_{i}(1,0) .
\end{aligned}
$$

For the specific parametric formulation of Example II, the two teams' optimal actions at both stages, under either regime $n=2$ or $n=3$, are given in the text. Using that, and the foregoing definitions for the expected number of goals and the probability that a match ends in a draw, one can express $y^{*}$ and $z^{*}$ as functions of the parameters $\kappa$ and $\ell$. It is then possible to verify that, for the parametric domain of interest derived in the text, that is, $0<\kappa \leq 1 / 4$ and $2 \kappa \leq \ell \leq 1-2 \kappa$, the claim results follow, that is, $\left.y^{*}\right|_{n=3}>\left.y^{*}\right|_{n=2}$ and $\left.z^{*}\right|_{n=3}<\left.z^{*}\right|_{n=2}$ 